\title{
Hepatic artery aneurysm rupture presenting as an upper GI bleed: A case report
}

\author{
M Wayne, C Wong, J Kim, B Gandolfi, D Hudesman, R Grossi
}

\begin{abstract}
Introduction: Hepatic artery aneurysms (HAAs) are a rare but a clinically important phenomenon. The natural history of HAA is poorly understood, however, it is suggested that mortality following spontaneous rupture is as high as 40\% [1]. Statistics such as these sanction an aggressive approach to the management of the HAA, whenever detected. Case Report: We present a case of a 42-year-old old male who presented with two episodes of hematemesis. He underwent esophagogastroduodenoscopy (EGD) twice with control of bleeding, only to rebleed, and become unstable. A computed tomography (CT) angiogram demonstrated a hepatic artery aneurysm, which appeared to involve the duodenal bulb. The patient was taken emergently to the operating room for surgical repair of the hepatic artery aneurysm. Conclusion: Hepatic artery aneurysms are a rare entity and even less common cause of gastro-intestinal bleeding.
\end{abstract}

Keywords: Bleeding, Duodenum, Hepatic, Artery

$$
* * * * * * * * *
$$

M Wayne ${ }^{1}$, C Wong ${ }^{1}$, J Kim ${ }^{1}$, B Gandolfi ${ }^{1}$, D Hudesman ${ }^{1}$, R Grossi ${ }^{1}$

Affiliations: ${ }^{1}$ The Department of Surgery and Department of GI Medicine, Beth Israel Medical Center, New York, NY USA.

Corresponding Author: Dr. Michael Wayne, 37 Union Square West, 4th floor, NY, NY 10003, USA; Ph: 212-6046822; Fax: 212-604-3383; Email: waynedocny@yahoo.com

Received: 27 March 2012

Accepted: 16 April 2012

Published: 02 June 2012
Wayne M, Wong C, Kim J, Gandolfi B, Hudesman D, Grossi R. Hepatic artery aneurysm rupture presenting as an upper GI bleed: A case report. International Journal of Hepathobiliary and Pancreatic Diseases 2012;2:15-19.

Article ID:100006IJHPDMW2012

$$
* * * * * * * * *
$$

doi:10.5348/ijhpd-2012-6-CR-4

\section{INTRODUCTION}

Hepatic artery aneurysms (HAAs) are a rare but a clinically important phenomenon. Hepatic artery aneurysms are hard to diagnose and have very few localizing symptoms. Approximately 40\% of these aneurysms will rupture, most commonly with either fistulization to the common bile duct causing hemobilia, or with rupture into the abdominal cavity causing hemoperitoneum [1].

\section{CASE REPORT}

A 42-year-old man with a past medical history significant for alcohol abuse, gastritis, osteoarthritis of bilateral knees and hypertension, presented with two episodes of hematemesis (reportedly two cups full of fresh blood) a day prior to admission. The patient noted multiple episodes of melena approximately three months prior to the episodes of hematemesis. During the last three months he had been drinking heavily and consuming about $200 \mathrm{mg}$ per day of indomethacin for bilateral osteoarthritis of the knee. The patient had a normal esophagogastroduodenoscopy, colonoscopy and bleeding scan two weeks prior to admission to our institution at a nearby medical center. Upon discharge from that institution, his hemoglobin was $7 \mathrm{~g} / \mathrm{dl}$. His 
home medications included indomethacin and prevacid. However, he stopped taking prevacid after he noted that he had an "irregular heartbeat" despite his gastroenterologist's advice to continue his prevacid.

The gastroenterology team was called for recurrent hematemesis. The patient's hemoglobin had dropped down to $5.7 \mathrm{~g} / \mathrm{dl}$ with a hematocrit of $18.4 \%$. The patient was tachycardic. He was immediately brought down for esophagogastroduodenoscopy under monitored anesthesia care. The entire esophagus was normal. There was fresh blood in the stomach but no gross lesions were noted. However, upon reaching the duodenal bulb a small, two mm, oozing erythematous, indented lesion was noted (Figure 1). Two hemostatic clips were successfully placed on the lesion (Figure 2). Bleeding ceased after therapy. The scope was withdrawn. Throughout the entire procedure, the patient's vital signs remained stable and the patient tolerated the procedure well. The initial diagnosis was a Dieulefoy's lesion and the patient after successful hemostasis was sent to the intensive care unit for furthering monitoring and management. Nexium drip was resumed and two units of typed packed red blood cells were ordered and given.

Two hours after the initial therapeutic esophagogastroduodenoscopy, the intensive care unit called us stating that hematemesis had once again resumed. The patient vomited approximately $300 \mathrm{ml}$ of bright red blood. The mobile endoscopic station was brought to the bedside and esophagogastroduodenoscopy was repeated under monitored anesthesia care to investigate the etiology of this recurrent bleed. The patient was again tachycardic but alert. He denied any abdominal pain. The endoscope was passed to the duodenal bulb where the same lesion was noted with oozing around the two intact hemostatic clips. Five $\mathrm{ml}$ of 1:10,000 solution of epinephrine was injected to the surrounding area and superfical coagulation using the ConMed Beamer (ConMed, USA) at 20 watts was applied to the site. Again hemostasis was achieved. The surgical service was also consulted. A CT angiogram of the abdomen and pelvis was ordered to investigate the recurrent hematemesis.

The CT angiogram revealed a large $3.7 \mathrm{~cm}$ pseudoaneurysm surrounding the proper hepatic and common hepatic arteries, extending into the proximal left and right hepatic arteries (Figures 3-6). The aneurysm sac abutted the posterior margin of the duodenal bulb and was visible endoscopically, where there was a clip projecting into the duodenal lumen. The patient was subsequently transferred to the surgical ICU and surgery was planned.

The decision was made to take the patient emergently to the operating room for definitive repair. An exploratory laparotomy was performed and control of the right and left hepatic arteries as well as the celiac trunk was attained. The aneurysmal sac was opened and repaired with a six $\mathrm{mm}$ ringed gore-tex graft. The aneurysm sac was closed over the graft. The duodenum was mobilized and dissected free of the hepatic artery aneurysm. The duodenal defect was closed primarily and a tongue of omentum was used to reinforce the repair. The patient tolerated the procedure well and was transferred to the intensive care unit.

The patient was transfused with two units of packed red blood cells to achieve a hemoglobin greater than 7 $\mathrm{g} / \mathrm{dl}$, and slowly returned to normal activity. The patient was discharged from the hospital in good condition seven days after his operation.

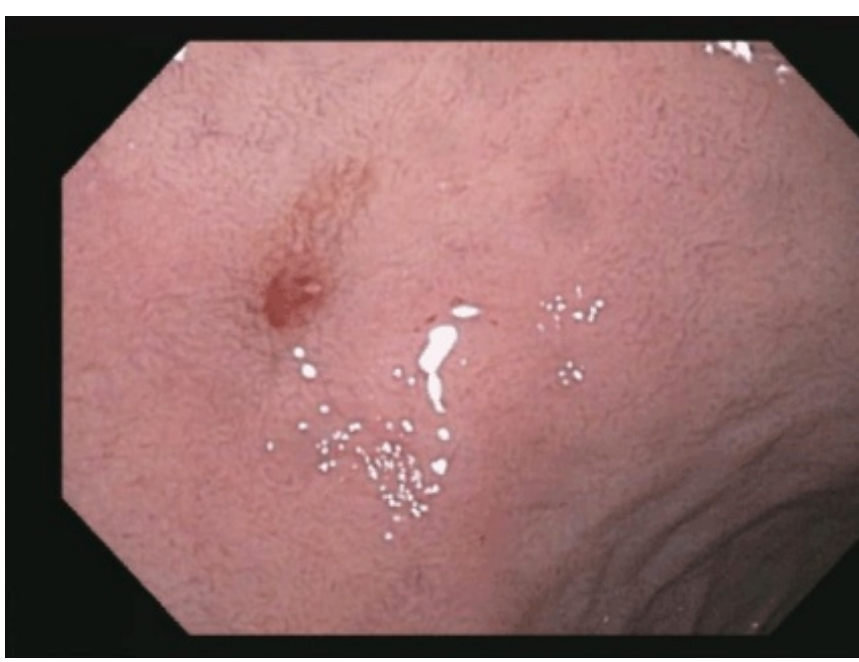

Figure 1: EGD appearance of bleeding source in duodenal bulb.

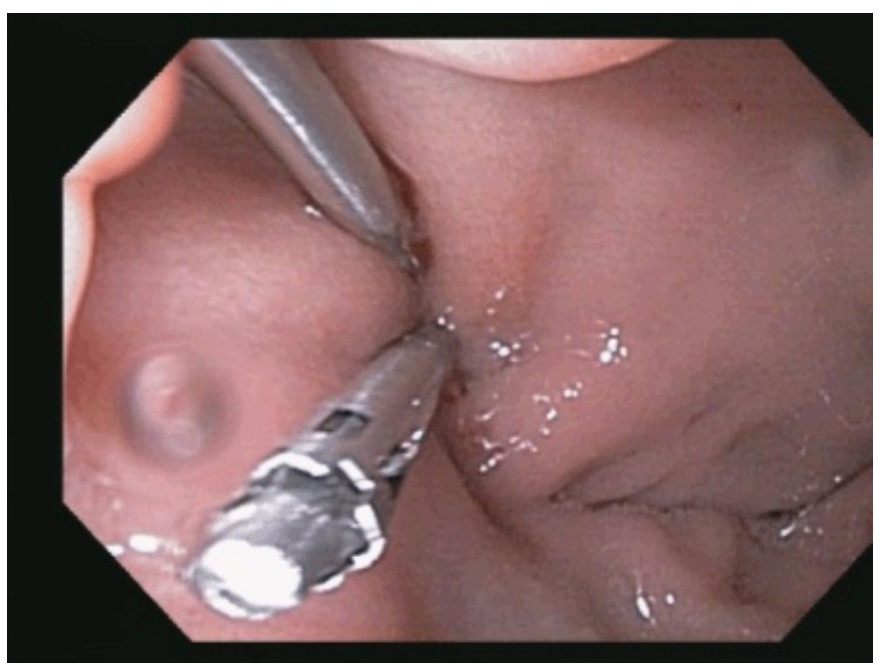

Figure 2: Duodenal bulb lesion after clipping of bleeding lesion.

\section{DISCUSSION}

Hepatic artery aneurysms (HAAs) are a rare but a clinically important phenomenon. A review of the literature between 1985 and 1995 showed that the HAA had surpassed splenic artery aneurysm (SAA) as the most frequently reported visceral artery aneurysm [2]. This recent trend is thought to be due to the proliferation of centres performing invasive diagnostic and therapeutic hepatobiliary procedures, many of 


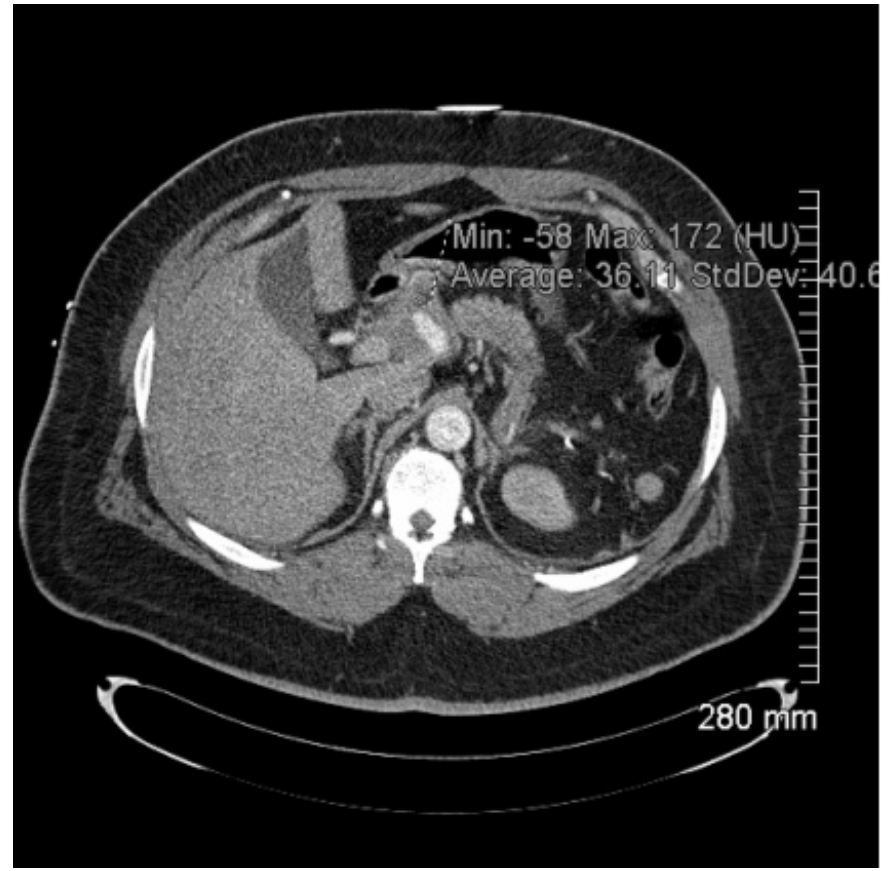

Figure 3: CT image of hepatic artery aneurysm.

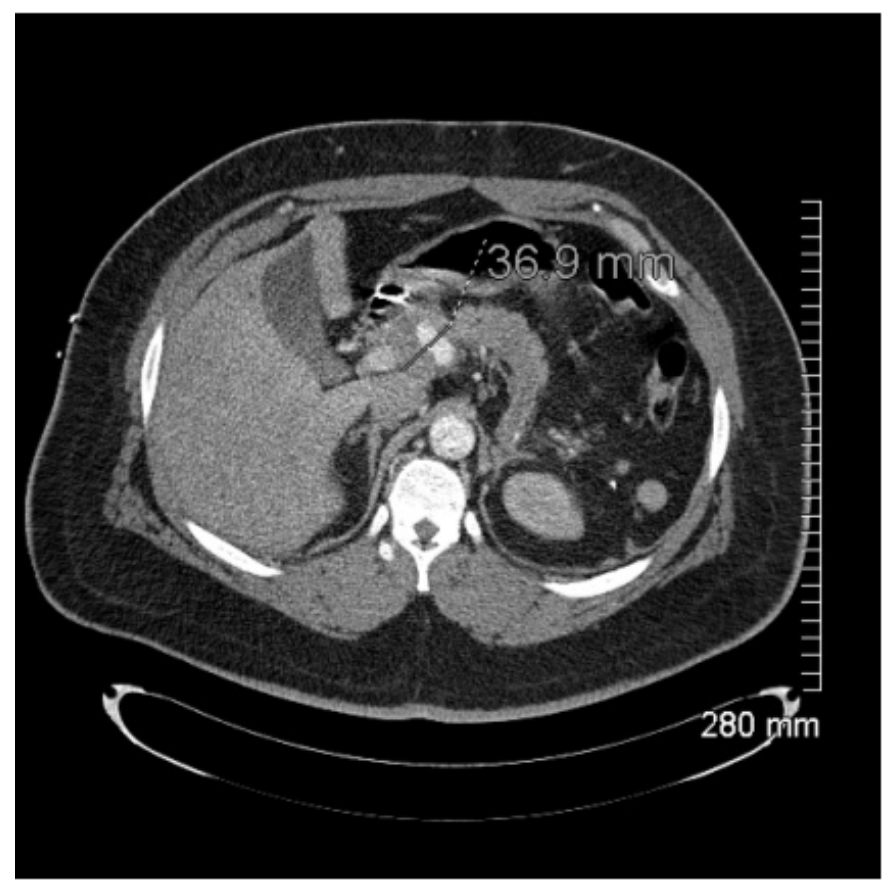

Figure 4: CT image of hepatic artery aneurysm.

which have hepatic artery pseudo-aneurysm formation as a recognised complication. The natural history of HAA is poorly understood, however, it is suggested that mortality following spontaneous rupture is as high as 40\% [1]. Statistics such as these sanction an aggressive approach to the management of the HAA, whenever detected.

Hepatic artery aneurysms are hard to diagnose and have very few localizing symptoms. Approximately 40\%

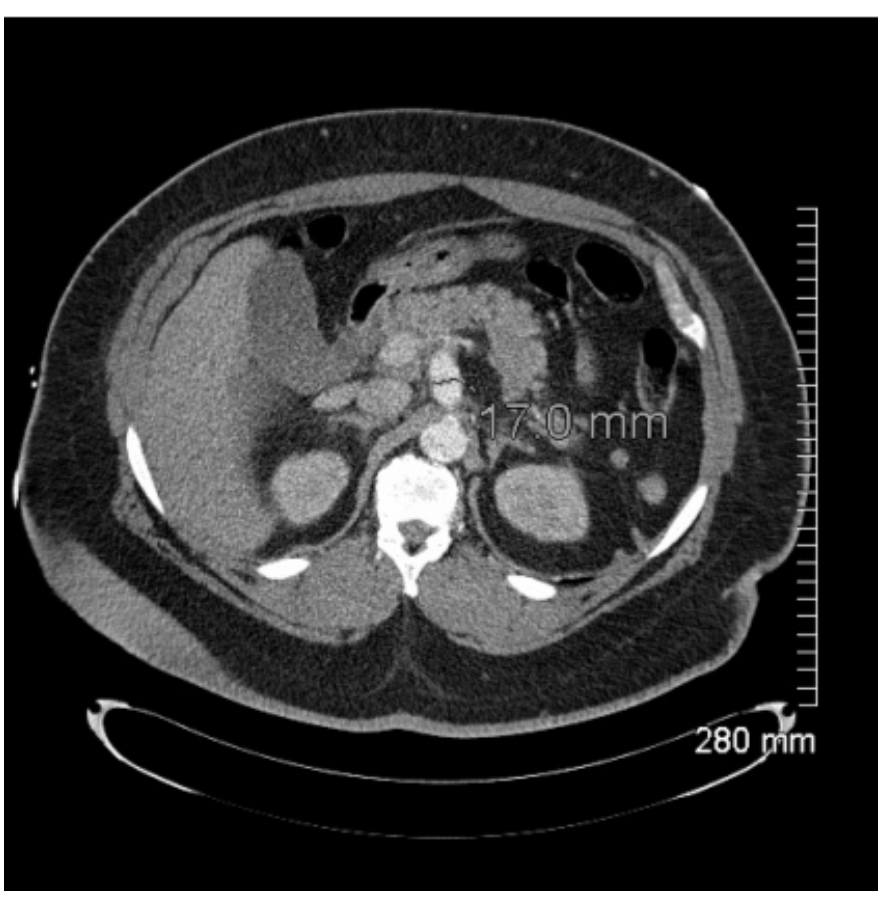

Figure 5: CT image of hepatic artery aneurysm.

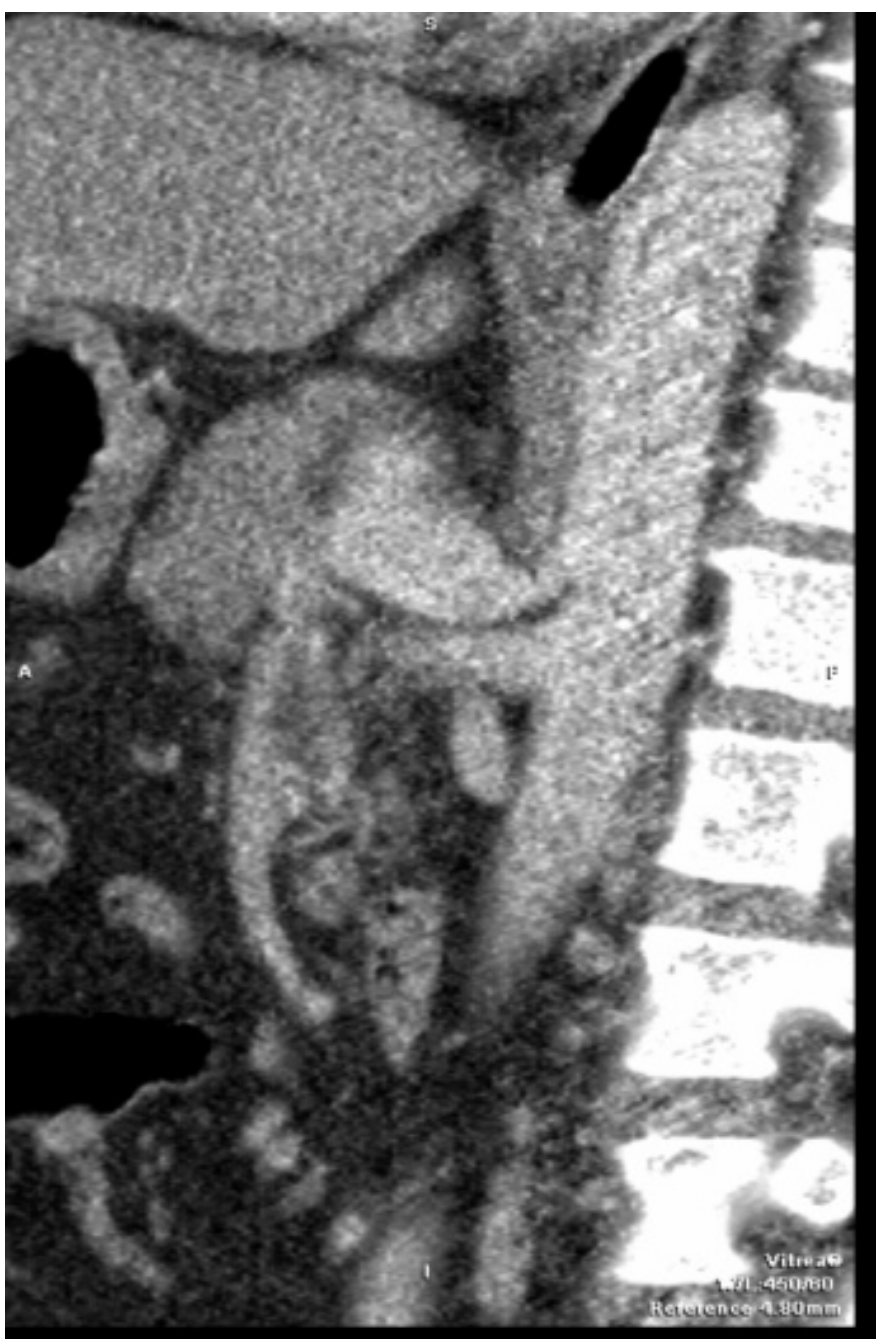

Figure 6: CT image of hepatic artery aneurysm. 
of these aneurysms will rupture, most commonly with either fistulization to the common bile duct causing hemobilia, or with rupture into the abdominal cavity causing hemoperitoneum [1].

Due to the high spontaneous rupture rate, hepatic artery aneurysms greater than two $\mathrm{cm}$ diameter must usually be treated when the diagnosis is confirmed [ 1 , 3]. Recently, non-surgical interventions such as coil embolization and stented graft insertion into the aneurysm sac is being more frequent applied in treatment of visceral artery aneurysm [3-5].

To our knowledge, only two hepatic artery aneurysm-duodenal fistulas are reported in the literature. In the first case, a severe case of cholecystitis is believed to have caused the development of a right hepatic artery aneurysm and the subsequent fistula. In the second case, the patient presented with an upper gastro-intestinal bleed, as our patient did. Both patients in the previous reports underwent laparotomy but it was not possible to attain control of the hepatic artery and eventually both aneurysms were repaired using endovascular techniques. In the case of right hepatic artery aneurysm, the right hepatic artery was embolized without any sequelae. In the common hepatic artery aneurysm, a bare metal stent was used to traverse the lesion and the aneurysmal sac was embolized using fibrin glue. Both of the above approaches have to be done with caution. A well known complication of hepatic artery embolization is loss of liver parenchyma and hepatic abscess formation. However, in high risk patients, this procedure may be the best alternative. The current literature supporting the use of bare metal stents for aneurysmal repair consists of only a few successful case reports and thus should only be attempted as a last resort. In our case, control of the hepatic artery was attained and repaired intraoperatively. Endoscopic hemostatic clips were placed on the bleeding area prior to the operative procedure. This technique for the management of gastrointestinal bleeds is becoming more common but should be executed with extreme caution, especially in cases where anatomy predisposes to an underlying vascular etiology.

\section{CONCLUSION}

Hepatic artery aneurysms are a rare entity and even less common cause of gastro-intestinal bleeding. Although no hepatic artery-duodenal fistula had been described prior to 2000 in the literature, this marks the third such presentation of this disease since that time. As our diagnostic modalities become more sensitive, we should expect more such cases as an etiology of massive upper gastrointestinal bleeds arising from the duodenum. These fistulas are surgical emergencies and generally require a laparotomy to control the duodenal component of the fistula and either open aneurysmectomy or endovascular repair of the vascular aneurysm.

\section{Author Contributions}

M Wayne - Substantial contributions to conception and design, Acquisition of data, Analysis and interpretation of data, Drafting the article, Revising it critically for important intellectual content, Final approval of the version to be published

C Wong - Substantial contributions to conception and design, Acquisition of data, Analysis and interpretation of data, Drafting the article, Revising it critically for important intellectual content, Final approval of the version to be published

J Kim - Substantial contributions to conception and design, Acquisition of data, Analysis and interpretation of data, Drafting the article, Revising it critically for important intellectual content, Final approval of the version to be published

B Gandolfi - Substantial contributions to conception and design, Acquisition of data, Analysis and interpretation of data, Drafting the article, Revising it critically for important intellectual content, Final approval of the version to be published

D Hudesman - Substantial contributions to conception and design, Acquisition of data, Analysis and interpretation of data, Drafting the article, Revising it critically for important intellectual content, Final approval of the version to be published

R Grossi - Substantial contributions to conception and design, Acquisition of data, Analysis and interpretation of data, Drafting the article, Revising it critically for important intellectual content, Final approval of the version to be published

\section{Guarantor}

The corresponding author is the guarantor of submission.

\section{Conflict of Interest}

Authors declare no conflict of interest.

\section{Copyright}

(C) M Wayne et al. 2012; This article is distributed under the terms of Creative Commons attribution 3.0 License which permits unrestricted use, distribution and reproduction in any means provided the original authors and original publisher are properly credited. (Please see www.ijhpd.com/copyright-policy.php for more information.)

\section{REFERENCES}

1. Abbas MA, Fowl RJ, Stone WM, Panneton JM, Oldenburg WA, Bower TC, Cherry KJ, Gloviczki P: Hepatic artery aneurysm: factors that predict complications. J Vasc Surg 2003;38:41-5.

2. Shanley CJ, Shah NL, Messina LM: Common splanchnic artery aneurysms: splenic hepatica and celiac. Ann Vasc Surg 1996;10:315.

3. Schick C, Ritter RG, Balzer JO, Thalhammer A, Vogl TJ. Hepatic artery aneurysm: treatment options. Eur Radiol 2004;14:157-9. 
4. Millonig G, Graziadei IW, Waldenberger P, Koenigsrainer A, Jaschke W, Vogel W. Percutaneous management of a hepatic artery aneurysm: bleeding after liver transplantation. Cardiovasc Intervent Radiol 2004;27(5):525-8.

5. Stambo GW, Guiney MJ, Cannella XF, Germain BF. Coil embolization of multiple hepatic artery aneurysms in a patient with undiagnosed polyarteritis nodosa. J Vasc Surg 2004;39(5):1122-4. 